\title{
Selection of Protective Helmets Based on the Scanning System
}

\author{
Konstantin KARAVAEV, Maxim TRUPHANOV \\ Elasizer Srl., Milan, Italy \\ https://doi.org/10.15221/19.081
}

\begin{abstract}
This publication presents the results of creating a service for individual on-line selection and customization of protective helmets for active sports and recreation. The service is based on the use of developed and patented hardware and software solutions that enable creation of three-dimensional shapes of the surface of a client's head, the inner surface of a helmet and the subsequent virtual combination of a client's head and helmet in order to calculate the vector of parameters of matching the anthropometric features of a client's head.

As a means of generating the primary data on the surface of a head, a mobile phone or a developed hardware scanner can be used. The service also provides an objective selection of the best fitting helmet with consideration of the individual subjective preferences of a client and analysis of a client's experience in using helmets. After selecting the best fitting commercially available helmet for a specific client, a helmet can be adapted using a specially designed inlay of a variable thickness in a helmet.

The adaption of a helmet ensures its maximum conformity to the shape of a head. Adaption is carried out on the basis of a special, inexpensive and easy-to-use helmet inlay. The thickness of the inlay is calculated automatically that ensures the best possible conformity with a customer, providing comfort and safety. The conducted experimental studies, as well as the reviews of customers who participated in testing the system, confirmed the desirability and accuracy of the selection of helmets based on the developed technology.
\end{abstract}

Keywords: headwear, helmet, measurement, customized, mass-produced, selection

\section{Introduction}

One of the key tasks in ensuring safety in active sports and recreation is the selection of a protective helmet. The helmet must be in firm contact with the surface of a head, yet create no discomfort for a client. For that purpose, the shape of the inner surface of a helmet must match the shape of a client's head.

The selection of a helmet for professional athletes is based on complex and expensive methods and components that are not available to the general public who are engaged in active sports, such as motorcycling, cycling, skiing and other.

For most amateur sporting participants selection of a helmet consists in a continuous physical fitting from a few up to a dozen articles in a store. Eventually, the most comfortable helmet is subjectively selected from the range of available items. Obviously, this traditional approach has several drawbacks. Firstly, it does not provide the maximum possible level of safety due to the subjectivity of the approach to the selection of a helmet. Secondly, it is time-consuming, uncomfortable and tedious both for a client and a shop assistant. Thirdly, it eliminates the guarantee that the helmet purchased online which was not tested physically will match the required size and shape.

\section{Method}

In the context of this work, we developed scanners for the surface of a head and the inner surface of a helmet, which collectively allow us to form three-dimensional models of a head and a helmet and provide an automatic selection of the most suitable helmets for clients. This ensures that not only the shape of a helmet is measured, but also the elasticity of the liner of the helmet is assessed in various points of contact with the surface of the head based on a unique patented technology. Based on the created and patented solutions, new methods and tools for virtual fitting and selection of the most suitable item of equipment were developed and experimentally tested.

This solution is unique because it provides a quick and accurate selection of safety equipment. The objectivity of a recommendation is achieved primarily by comparing a client's scan data with helmets. The recommendation can be specified with consideration of individual preferences of a client who already has a comfortable helmet. 
The technical solution is based on the use of the following key patented solutions:

- portable mobile scanner and software solution for scanning the inner surfaces of objects, helmets,

- technical solution for high-precision scanning of the surface of a head based on a stationary multi-sensor scanner of external surface of objects,

- mobile application which provides integration of the system and interaction with a client.

The key purpose of the developed solution is to increase the level of comfort and safety. Given the individual anthropometric characteristics of a head and subjective requirements, in addition to the recommendation of an existing helmet, quick and easy adaption of an existing helmet is possible.

The solution to the problem consists of the following main steps:

- creation of a database of helmets using the developed scanners; at the same time, the database stores not only the three-dimensional shape of the surfaces of helmets, but also a supplementary set of characteristics measured by means of the developed solution;

- creation of a three-dimensional model of the shape of a head using a scanner or mobile phone, as well as analyzing a client's preferences based on experience and formalized preferences (fig. 1);

- choosing the best fitting helmet among the range of mass-produced products and calculating the parameters of the adaption inlay, which will provide the maximum possible comfort and safety for a particular client while using this particular helmet;

- creation of the adaption inlay and fixing it inside a helmet.
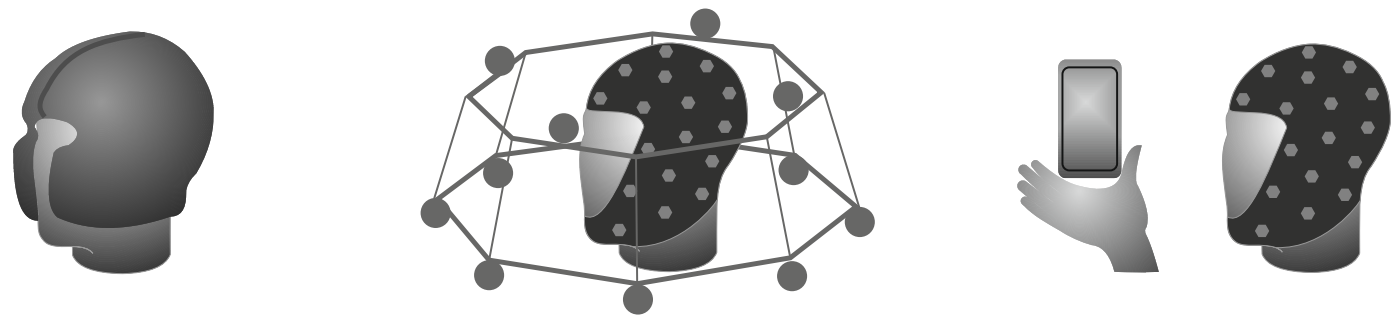

Figure 1. Scanning the shape of a head using a scanner or mobile phone

The adaption inlay is an insert that repeats the individual characteristics of the client's head.

When the elastic inlay is placed inside a helmet, a helmet is adapted for a specific client and ready for use.

Considering the proposed technical solutions, first of all, we note that scanning the shape of the surface of a head in the optical range is associated with the objective problem that a client has hair of various style and thicknesses. To eliminate this problem, an elastic contact coating in the form of a cap of universal size is put on the head for scanning. Graphic markers are applied on the front surface of the cap, thus increasing the accuracy of matching the points on the surface and calculation of the shape of the surface of a head in three-dimensional space.

To scan the head, a multisensor scanner can be applied based on several rigidly mounted cameras that make a single system or a mobile phone with a developed application. The primary data for calculation is the coordinates of the key points in two-dimensional space. All calculation in both cases is done in the cloud.

The multi-sensor scanner is designed as a spatial structure which contains 12 inexpensive digital cameras. This solution ensures the simultaneous reception of images of an object from all sides with a not more than 20 milliseconds difference in the time of reception of frames. The measurement accuracy, in this case, is from 0.5 to $1.2 \mathrm{~mm}$, depending on the part of a head.

This is the fastest, most convenient and most accurate way to scan.

It must be noted that without any modification this scanner can be used to scan legs and hands. 
In addition to a multi-sensor scanner and a mobile application, a head scan can be carried out using a flexible contact scanner, which is a unique patented solution in which instead of a single-use elastic coating with markers, a reusable flexible helmet is used to create physical prints of the scanned object. The device captures a print of the surface of a head by creating a vacuum inside the sealed sides of a helmet, after which the data on the surface of a head is already taken from the inner surface of the helmet.

This is also a quick way to measure the head, which additionally has the unique advantage of direct physical control of the contact and pressure force, taking into account the individual preferences of a client during imprinting. After receiving the print, it can be digitized by various scanning solutions. We use our own solution, which is based on the registration of images of the inner surface of a helmet with markers on the surface.

The mobile application for a smartphone used for scanning is the most convenient for public use. But at the same time, for now, it is the least accurate means of measuring the shape of the surface of a head.

The principle of operation of a mobile application is based on obtaining a small series of images of a head, calculating the parameters of key points on the surface of a head, transferring the received data to a cloud for computation, and then providing the user with the obtained scan results while selecting the best fitting helmet.

In addition to the means for obtaining a model of a three-dimensional surface of a head, it is necessary to consider a scanner for the inner surface of an object.

The scanner of the inner surface of an object (helmet or other item of protective equipment or orthopedic aids) is a portable device which consists of two cameras driven by a servomotor along a predetermined trajectory inside the scanned object (fig. 2). The scanner is positioned in such a way that the scanning elements (cameras and illumination means) are located inside the scanned object, after which the scanning process is initialized and a three-dimensional model of the scanned object is created.
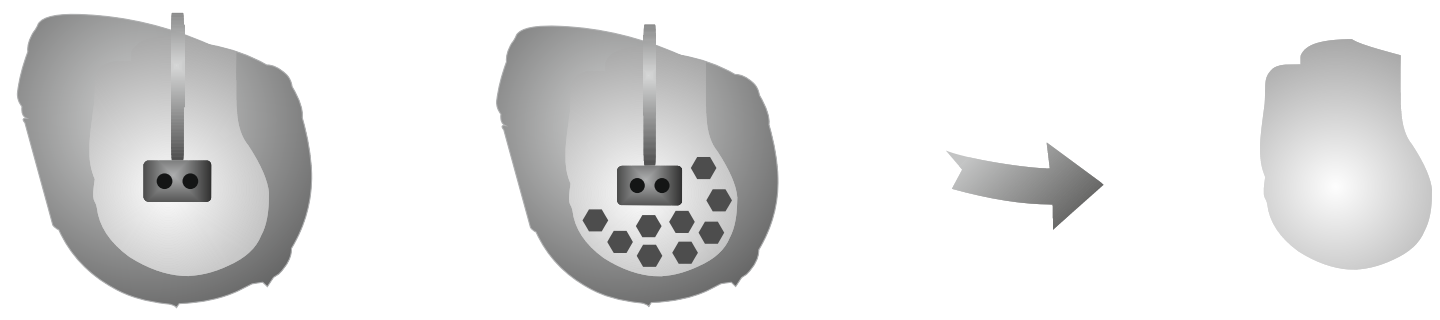

Figure 2. Inner surface scanning

The scanner provides a database of the internal surfaces of a helmet. The proposed algorithms for scanning the inner surface of a helmet allow to create an accurate map of the inner surface of a helmet, as well as a map of the firmness of the surface of a helmet. Moreover, these maps are created with consideration of subsequent positioning of the helmet on a head based on the analysis of the positions of key points, which then, along with the obtained three-dimensional models of the shape of a helmet, are used in the service of selecting the best fitting helmet and its adaption (fig. 3).
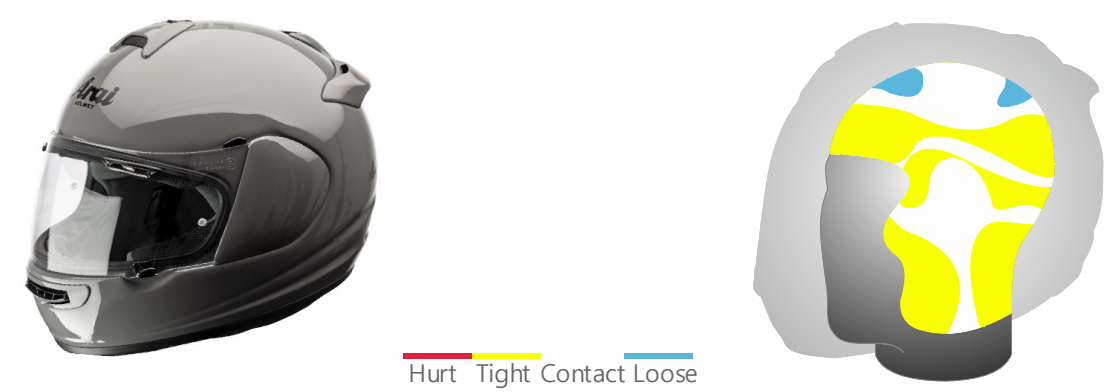

Figure 3. The color map illustrating the process of choosing the best fitting helmet 


\section{Conclusion and future work}

We described technical solutions that provide initial data for the selection and adaption of a helmet, namely, the developed tools that provide the creation of the shape of the surface of a head and the creation of the shape of the surface of a helmet.

The next step is the selection of the closest helmet and its adaption by means of proposed helmet insert that we developed.

Customization of a helmet is based on calculating the closeness of the surface of a head to the surface of a helmet at predetermined key points. After receiving such data during the calculation in the cloud, the client can independently use our adaption insert to form an additional surface placed inside the helmet of the thickness specified at the required points. After that, the helmet is ready for use.

Thus, the technology and technical solutions for the fast, accurate, cheap and convenient process of customization of mass-produced helmets by a client are presented. Distinctive advantages of the developed solution are the following: high speed, availability, scalability and practical applicability in specific products.

The developed solution allows to provide a quick and convenient selection of a protective helmet for professional and amateur athletes with consideration of objective characteristics and to increase the level of comfort and safety. 